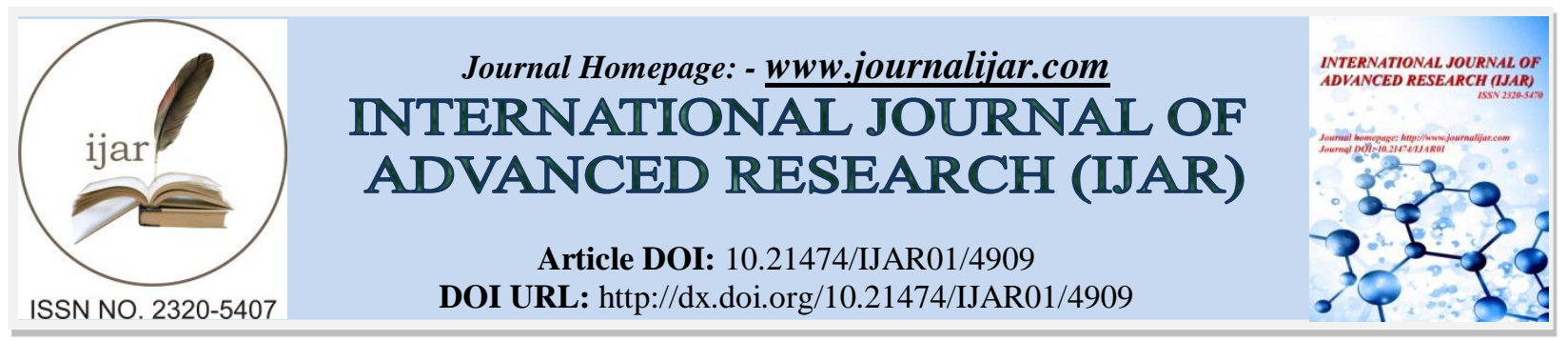

RESEARCH ARTICLE

\title{
MINIMALLY INVASIVE TECHNIQUES FOR REGENERATIVE PERIODONTAL THERAPY.
}

Dr. Anamika Sharma ${ }^{1}$ and Dr. Himani Sharma ${ }^{2}$.

1. Professor and Head, Department of Periodontology, Subharti Dental College and Hospital, Meerut. India.

2. Post graduate student Department of Periodontology, Subharti Dental College and Hospital, Meerut. India.

\section{Manuscript Info}

\section{Manuscript History}

Received: 21 May 2017

Final Accepted: 23 June 2017

Published: July 2017

Key words:-

Minimally invasive surgery,

periodontitis, regenerative procedures

\section{Abstract}

With the advancement in the field of periodontology various techniques have been evolved to minimize the post-operative discomfort and morbidity occurring after periodontal surgical procedures. Periodontal diseases are a heterogeneous group of diseases, which are multifactorial and are characterized by inflammation and the subsequent destruction of the tooth supporting structures. Conventional periodontal regenerative procedures involved reflection of large periodontal flaps for access to achieve improved clinical outcomes of periodontally compromised teeth. However, minimally invasive surgical techniques utilizes magnification devices to advanced surgical instruments and modified procedures, to make the surgical procedures effortless and causing minimal soft tissue trauma thus, proving to be advantageous over the conventional therapy. This article reviews various minimally invasive procedures and highlights the advantages of minimally invasive surgical and nonsurgical therapy.

Copy Right, IJAR, 2017,. All rights reserved.

\section{Introduction:-}

Periodontitis is a chronic multifactorial disease, evoked mainly by accumulation of dental plaque, forming a biofilm around the tooth, inducing inflammation cascade in the periodontal soft and hard tissues, breaking down the components of the periodontium and leading to tooth loss. ${ }^{1}$

The goal of periodontal therapy is, to create and maintain good oral health, function, and aesthetics by arresting periodontal disease progression, along with the regeneration of structures lost to disease. The appropriate conventional non-surgical and surgical approaches along with regenerative techniques continue to offer reliable methods to reduce periodontal pockets, and attain improved periodontal architecture.

For the past 150 years, conventional surgical techniques with extensive flap procedures were used to treat generalized periodontal disease, and to gain access to the underlying diseased tissues were used. ${ }^{2}$ These procedures lead to loss of gingival tissue, causing unwanted changes in gingival contour and height leading to undesirable esthetic results, root sensitivity, bone damage, and making mortality and morbidity an unavoidable part. ${ }^{3}$

However, in the early 1980s, a new attitude began to develop, where less invasive techniques for surgical intervention were supported, as these techniques resulted in reduced risk of complications, along with decreased mortality and morbidity rate and better esthetic results. 
Also, with awareness about non-surgical periodontal therapy for the treatment of periodontitis, the probability to treat isolated rather than generalized periodontal defects increased.

So, the clinicians shifted their focus to develop, analyze, and employ the surgical procedures, aimed at greater and more predictable regeneration of periodontal tissues and functional attachment close to their original level, without causing much trauma to the tissues, in clinical practice.

Wickham and Fitzpatric ${ }^{4}$ described these surgeries as "Minimally Invasive Surgery (MIS)". "These are smaller and more precise surgical procedures, utilizing small incisions, utilizing operating microscopes and microsurgical instruments and materials, to achieve same surgical end point as that of conventional surgical techniques i.e. probing depth reduction and regeneration of bone and supporting tissues, but with minimal negative changes in soft tissue contours, and better esthetic results."

In 1993 Hunter and Sackier" refined the concept of MIS, as "the ability to miniaturize our eyes and extend our hand to perform microscopic and macroscopic operations in places that could previously be reached only by large incisions".

\section{Characteristics of MIS:-}

- Less operative trauma than an equivalent invasive procedure.

- Less pain and scarring.

- Speedy recovery.

- Reduces post-surgical complications.

Minimally invasive periodontal surgeries (MIPS) were described in 1998, by Tibbets and Shanelec. These were precise surgical techniques, concentrating on soft tissue regeneration and augmentation procedures with surgical operating microscope, and aimed to produce minimal wounds, minimal flap reflection and gentle handling of soft and hard tissues during periodontal surgeries. ${ }^{6}$

Cortellini and Tonetti $2009,{ }^{7}$ with Minimally Invasive Surgical Techniques (MIST) stressed upon primary wound closure, along with wound and blood clot stability and further enforced with Modified-Minimally Invasive Surgical Technique (M-MIST), to additionally incorporate the concept of space provision for regeneration.

The authors also suggested the use of MIPS in combination with bone grafts covered with a Bio-Resorbable Vicryl Mesh. The ideal site for bone grafting using MIPS is isolated, interproximal, defect not extending beyond the interproximal or periodontal defect bordering an edentulous area. The defect extending to the buccal or lingual from the interproximal area becomes a less ideal site and generalized horizontal bone loss or multiple interconnected vertical defects are the contraindication for MIPS. ${ }^{8}$

\section{Features of minimally invasive periodontal surgery:-}

\section{Incisions:-}

The incisions should conserve as much of the soft tissue as possible. Initial intrasulcular incision, is made on the teeth neighboring the defect, and followed by single horizontal incision, 2-3 mm apical from the crest of the papilla, usually in the palatal/lingual aspect to cover the grafted site and to preserve shape of papilla for better esthetics.

As stated by Allen ${ }^{9}$ in 1984, the Tunnel Technique (TT), is considered to be an important element of MIPS. In TT, after inrasulcular incision, subperiosteal tunnel is prepared through defect areas to allow the transplantation of subepithelial connective tissue graft (SECTG) which is extracted by single incision technique. ${ }^{1}$

\section{Flap Elevation:-}

Papilla is sharply dissected by means of Orbans knife, reshaped to one third or one forth to its original size to minimize trauma to the flap and preserving blood supply, for better healing and minimization of postoperative soft tissue changes.

Blunt dissection leads to, blanching of the reflected tissues causing darkened bruised appearance of the reflected tissues at the time of flap closure, leading to flattening of papilla, interproximal cratering, and loss of soft tissue height post surgically. 
Also, periosteum can be left on bone surface, by reflecting split thickness flap, to achieve a coronally tension free flap reflection and less post-operative bone loss and edema. ${ }^{11}$

\section{Papilla Preservation:-}

Along with the various surgical techniques developed to prevent the apical migration of the gingival margin during the treatment of bony defects in the anterior or esthetic zone Papilla Preservation Technique proposed by Takei et $\mathrm{al}^{12}$ is one where, no incisions were given through the interdental papilla.

The flap design included sulcular incisions around each adjacent tooth buccally and lingually/palatally, along with a semi-lunar incision across each interdental papilla dipping apically along the lines angles of the tooth such that the incision line is $5 \mathrm{~mm}$ away from the gingival margin.

The interdental tissue is then dissected and elevated intact with facial flap, repositioned, and sutured palatally. Cortellini et $\mathrm{al}^{13}$ gave a modification of this technique. In which a horizontal incision is performed on the buccal papillary tissue at the base of the papilla, where the interdental space is wide $(2 \mathrm{~mm})$. In narrow interdental space Simplified Papilla Preservation Flap (SPPF) is performed. ${ }^{14}$

\section{Defect debridement \& Placement of Grafting Material:-}

After flap reflection, the granulation tissue is sharply dissected from papillary supracrestal connective tissue within the osseous defect with blade and carved away with mini-curettes and sonic/ultrasonic instruments.

Final root planing is done with finishing burs and root conditioning is done with citric acid or EDTA.

A modified amalgam gun with a curved tip is used to place regenerative materials, e.g. amelogenins, growth factors, EMD, autografts, allografts - decalcified freeze dried bone allograft mixed with tetracycline hydrochloride. An unsutured vicryl mesh can be placed to hold the graft.

\section{Suturing Technique:-}

The use of microsuturing is an important element of MIPS. ${ }^{11}$ It prevents the collapse of gingiva and enhances optimal wound adaptation. Monofilament sutures are preferred whereas, sutures from 6-0 to 8-0 are mandatory, as they are less traumatic, and persists less chances of wound contamination. In anterior areas, vertical mattress sutures are recommended, whereas in posterior premolar and molar region modified matrix suture is used.

\section{Armamentarium:-}

Microscope- With magnification and illumination with devices like Perioscope, the visibility of the surgical field and the control of surgical instruments are improved, making the procedure short and less invasive. The intraoperative and post-operative morbidity is reduced thus amplified healing is achieved.

Microinstrumentation - Miniature instruments like microspatorium, bone scraper, papilla elevatorium, microscalpel holder, needle holder, microscissor, dental microforceps make MIST less traumatic.

\section{Local Drug Delivery (LDD):-}

The use of LDD device as an adjunct to SRP in deep pockets where conventional mechanical therapy fails to reach has reported a statistically significant reduction of probing depth, attachment loss. Various drugs used as LDD are tetracycline, minocycline, chlorhexidine, doxycycline, $1 \%$ metformin gel etc. LDD has diminished the need for extensive periodontal surgical procedures and provide a localized diseased area for MIST. ${ }^{15}$

Lasers / Photodynamic Therapy:- Laser assisted periodontal therapy (LAFT) -It is more precise and targeted technique than traditional surgical method. Various studies have reported a significant improvement in probing depth, clinical attachment level and healing of the pocket with LAFT suggesting that laser is an effective minimally invasive surgical modality for treatment of moderate to advanced periodontal diseases. ${ }^{16}$

Photodynamic therapy (PDT) is an effective and innovative microbicidal method which involves the combination of a non-toxic dye (photosensitizer) and a visible light source. PDT has proven to be a useful adjunct to mechanical therapy as well as antibiotics in eliminating periopathogenic bacteria even in the site difficult to access. PDT is recognized as a treatment strategy which is both minimally invasive and minimally toxic. 
Thus, both these techniques decline the need for extensive periodontal surgical procedures and favors MIST.

\section{Minimally Invasive Implant surgery:-}

Precise surgical preparation of implant site is critical, to achieve primary stability and successful osseointegration. The conventional open flap technique for implant site preparation provides direct visual and instrumental access to the surgeon for the preparation of a good osteotomy site for primary stability. However, it also increases the chances of morbidity of the site and delays the postoperative recovery period when compared with less invasive methods.

Minimally invasive implant surgery, commonly referred to as "flapless" implant surgery, offers numerous advantages over conventional open flap technique.

\section{These advantages include:-}

- Preservation of circulation, soft-tissue architecture and hard-tissue volume.

- Decreased surgical time.

- Improved patient comfort and minimization of postsurgical morbidity.

However, an emphasis on proper case selection following detailed clinical and radiographic examination including CBCT, should be given to ensure the success of MIST with implants.

\section{Advantages of MIST:-}

- Reduction in Post-operative complications- such as edema and root hypersensitivity and side effects in the post-operative period is seen. ${ }^{17}$

- Reduction in post-operative healing period- Cortellini and Tonetti in $2009^{17}$ reported the achievement of primary closure of the flap during the early healing in $91 \%$ of cases whereas, $70 \%$ was seen following traditional papilla preservation flaps.

- Reduction in intraoperative pain or discomfort was seen in patients treated with MIST and EMD and only 30\% of the patients reported some moderate post-operative pain which disappeared within $26 \pm 17$ hours. ${ }^{18}$

\section{Limitations:-}

- Limited visibility in M-MIST makes management of the surgical field more critical.

- Difficulty in achieving access to the root surface through buccal window in deep palatal defects. ${ }^{18}$

- Technique Sensitive and requires specialized instruments and materials, which could be more expensive.

- Some procedures may take longer than usual, compared with conventional surgeries.

\section{Conclusion:-}

Minimally invasive surgery might be considered as the future in the field of periodontal regeneration. Several clinical trials have shown limited morbidity and enhanced healing during surgical procedure and in post-operative healing period with MIST. However, the success of MIST depends mainly on the case selection. Also, along with the use of specialized microinstruments and materials MIST requires specific training. Many studies assures the effectiveness of MIPS, however more studies are required to confirm the effectiveness of these techniques over traditional ones in periodontal surgery. 


\section{References:-}

1. Socransky SS, Haffajee AD. Dental biofilms: Difficult therapeutic targets. Periodontol 2000 2002;28.

2. Schluger S. Osseous resection - a basic principle in periodontal surgery. Oral Surg Oral Med Oral Pathol 1949;2:316-325.

3. Stephen KH, Martha EN, Claire MB. Long-term results of a minimally invasive surgical approach for bone grafting. J Periodontol 1999;70:1558-1563.

4. Wickham J, Fitzpatric JM. Minimally invasive surgery.Br J Surg 1990;77:721-722.

5. Hunter JG, Sackier JM. Minimally invasive high tech surgery: Into $21^{\text {st }}$ century. Minimally Invasive Surgery. New York:McGraw-Hill;1993;3-6

6. Tibbets LS, Shanelec DA. An overview of periodontal microsurgery. Current Opin Periodontol 1994;1:183-187

7. Cortellini P, Tonetti MS. Improved wound stability with a modified minimally invasive surgical technique in the regenerative treatment of isolated interdental intrabony defects. J Clin Periodontol 2009;36:157-63.

8. Harrel SK. A minimally invasive surgical approach for periodontal regeneration: surgical technique and observations. J Periodontol 1999:70:1547-57.

9. Allen EP. Advances in mucogingival surgery. Tex Dent J 1984;101:26-30.

10. Hurzeler MB, Weng D. A single-incision technique to harvest subepithelial connective tissue grafts from the palate. Int J Periodontics Restorative Dent 1999;19:279-87.

11. Gassmann G, Grimm WD. Minimal-invasive regenerative und plastisch-rekonstruktive Parodontalchirurgie. Dent Implant Pariodontol 2006;10:90-7.

12. Takei HH, Han TJ, Carranza FA Jr, Kenney EB, Lekovic V. Flap technique for periodontal bone implants: Papilla preservation technique. J Periodontol 1985;56:204-10.

13. Cortellini P, Prato GP, Tonetti MS. The modified papilla preservation technique. A new surgical approach for interproximal regenerative procedures. J Periodontol 1995;66:261-6.

14. Cortellini P, Prato GP, Tonetti MS. The simplified papilla preservation flap: A novel surgical approach for the management of soft tissues in regenerative procedures. Int J Periodontics Restorative Dent 1999;19:589-99.

15. Meinberg TA, Barnes CM, Dunning DG, et al. Comparison of conventional periodontal maintenance versus scaling and root planing with subgingival minocycline. J Periodontol. 2002:73:167-172.

16. Mizutani K, Aoki A, Coluzzi D, Yukna R, Wang CY, Pavlic V, Izumi Y. Lasers in minimally invasive periodontal and peri-implant therapy. Periodontol 2000. 2016;71(1):185-212.

17. Cortellini P, Nieri M, Prato GP, Tonetti MS. Single minimally invasive surgical technique with an enamel matrix derivative to treat multiple adjacent intra-bony defects: Clinical outcomes and patient morbidity. J Clin Periodontol 2008;35:605-13.

18. Cortellini P, Tonetti MS. Improved wound stability with a modified minimally invasive surgical technique in the regenerative treatment of isolated interdental intrabony defects. J Clin Periodontol 2009;36:157-63. 